\title{
CLINICO-BACTERIOLOGICAL PROFILE OF LATE ONSET SEPTICEMIA IN M. Y. H. NURSERY
}

Dhanuram Mandavi ${ }^{1}$, Nilesh Jain ${ }^{2}$

${ }^{1}$ Resident Doctor, Department of Paediatrics, M. G. M. Government Medical College, Indore, Madhya Pradesh.

${ }^{2}$ Assistant Professor, Department of Paediatrics, M. G. M. Government Medical College, Indore, Madhya Pradesh.

\section{ABSTRACT}

\section{BACKGROUND}

Septicaemia plays a major role in morbidity and mortality of neonates. This is one of five major causes of death in neonates. Late onset septicaemia remains a significant cause of morbidity and mortality in neonates, more so in developing countries.

\section{AIM OF STUDY}

To study the clinical pattern, risk factors, bacteriological profile, antibiotic sensitivity pattern and mortality rate in late onset septicaemia.

\section{STUDY DESIGN}

Prospective observational study.

\section{SETTING}

Nursery of M.Y. Hospital, MGM Medical College, Indore (M. P.)

\section{PARTICIPANTS}

One hundred newborns delivered and admitted in nursery of M.Y. Hospital, Indore, were taken up for study.

\section{METHOD}

Cases followed longitudinally regularly till discharge or death. The symptoms and sign closely observed and recorded and risk factors predisposing to late onset sepsis were analysed. Investigation done for sepsis profile, e.g. TLC, DLC, blood culture, CSF routine microscopy and culture and antibiotic sensitivity.

\section{RESULTS}

In present study, $78 \%$ cases belong to $1-2 \mathrm{~kg}$ group. Majority of cases (89\%) were become culture positive between 3rd-7th day of life. Prematurity at birth was most common indication of admission, $78 \%$ of cases followed by respiratory distress in $25 \%$. Neonates show following clinical features, lethargy $89 \%$, refusal to feed $84 \%$, jaundice $60 \%$, hypothermia $63 \%$ of the cases; 115 cultures were obtained from 100 patients showed that most common organism isolated was Staph aureus in $48.5 \%$ cases followed by E. Coli (30.8\%) and Klebsiella pneumoniae (27.9\%). Out of 100, 67\% cases were discharged and 25\% mortality was noted. In the study, maximum deaths were occurred in 5-12 days of life.

\section{CONCLUSION}

Pre-term and low birth weight babies are commonly affected, they became septic commonly in first week of life. Lethargy, refusal of feed and hypothermia are the common presentation of septicemia. Staph is the most common pathogen followed by E. coli in our study. Mortality rate in nosocomial septicemia is $25 \%$. Mortality rate is higher in female babies as compared to male babies. Mortality rate is high in LBW babies.

\section{KEYWORDS}

Septicemia, Blood Culture, Neonate.

HOW TO CITE THIS ARTICLE: Dhanuram Mandavi, Nilesh Jain. “Clinico-Bacteriological Profile of Late Onset Septicemia in M. Y. H. Nursery." Journal of Evolution of Medical and Dental Sciences 2015; Vol. 4, Issue 100, December 14; Page: 16520-16524, DOI: $10.14260 /$ jemds/2015/2458

\section{INTRODUCTION}

Septicemia plays a major role in morbidity and mortality of neonates. This is one of five major causes of death in neonates. Late onset sepsis is the nursery acquired septicemia, occurs

Financial or Other, Competing Interest: None.

Submission 21-11-2015, Peer Review 23-11-2015,

Acceptance 08-12-2015, Published 12-12-2015.

Corresponding Author:

Dr. Nilesh Jain,

G-79, M.I.G. Colony,

Near Bhartiya Academy Indore,

Madhya Pradesh-452011.

E-mail: nileshkalashdhar@gmail.com

DOI:10.14260/jemds/2015/2458 usually after 72 hours of life. In late onset infection, the organism first colonizes the baby and only later invades to cause sepsis. Sepsis screen was considered to be positive if at least two tests were positive (Abnormal leucocyte $(<5000$ $>20,000)$, I:T ratio $>0.12$, micro ESR $(15 \mathrm{~mm}$ at $1 \mathrm{hr}$.) and positive CRP.

Late onset septicemia remains a significant of morbidity and mortality in neonates, more so in developing countries. This may be due to delivery and postnatal followup in an unclean environment and non-adherence to aseptic measures, which increases the chance of contamination with infective organisms. An early diagnosis and appropriate antibiotic therapy leads to better survival rates in septicemia. However, 
antibiotics can be started early if we pick up septicaemia earlier by clinical evaluation. But starting antibiotics without documenting sepsis by blood culture and other haematological profile (Abnormal leucocyte count, thrombocytopenia, elevated micro increased ESR, increased I:T ratio and abnormality in CSF routine microscopy and culture) can result in administration of antibiotics more often babies without septicemia than babies with septicemia. Keeping this thing in mind it is important to study the clinical profile, bacteriological profile and risk factors in detail.

\section{MATERIAL AND METHODS}

Type of Study-Prospective observational study.

Source of Data: A total of 100 newborns delivered and admitted in nursery of M. Y. Hospital, Indore, were taken up for study. Newborn should be delivered and admitted in M. Y. Hospital nursery for at least $72 \mathrm{hrs}$ are included in study. Only those cases are selected who develops signs and symptoms of sepsis after $72 \mathrm{hrs}$ of birth.

\section{METHOD}

A detailed maternal history was taken followed by examination of the neonate and the cases followed longitudinally regularly till discharge or death. The symptoms and sign closely observed and recorded on case sheet as well as on proforma, specially designed for the purpose. The various symptoms that were inquired were fever, off feed, loose motion, convulsions, respiratory distress, abdominal distension, vomiting, keeping dull.

The various clinical signs looked for were hypothermia, crying, activity, rooting, sucking, cyanosis, pallor, jaundice and bleeding from any site.

Various risk factors predisposing to late onset sepsis were analysed in detail for each baby, e.g. birth weight, resuscitation at birth, gestational age, duration of stay, number of days for which peripheral vascular cannula was placed, etc.

\section{Exclusion Criteria}

1. Babies with PROM $>12$ hours.

2. Maternal fever.

3. Foul smelling liquor.

4. Onset of symptoms within 72 hours of life.

\section{Total Leukocyte Count and Differential Count Total Leukocyte Count}

The sample was taken in EDTA vial and 20 micro lit of sample was taken in pipette with $0.38 \mathrm{ml}$ of WBC diluting fluid, after waiting 5-10min Neubauer's was charged. Counting was done in four corner squares of chamber and the number obtained multiplied by 50 to get total leucocyte count.

\section{Differential Count}

For differential count a thin slide was prepared, allowed to dry, then staining was done with Leishman stain/Field's stain/Giemsa stain. The counting was done at the junction of tail and body part of the slide. Counting was done in oil immersion power of light microscopy. Counting was done in zig-zag fashion after dividing slides in 10x10 squares.

Counting for neutrophil was done for various forms depending on number of segment of nucleus. Cells were considered as immature when nucleus were seen as bilobed with two segments joining by broadband and more than four or five segmented nucleus neutrophils considered as mature. A ratio of $>0.12$ of immature to total cell count was considered as abnormal.

\section{Blood Culture}

Cultures were taken with full aseptic precautions. Child was placed in supine position and cleansed the site with $70 \%$ ethanol swab in one direction only, approx. $50 \mathrm{~mm}$ area is cleaned, then finally povidone iodine was applied and dried. The sample was taken by 22 -gauge needle; $2-3 \mathrm{ml}$ blood was drawn into the container of $25 \mathrm{ml}$ of liquid broth (Sodium polyanethol sulphonate), is a good anticoagulant, has added advantage of annulling the natural bacteriological action of blood. Liquid broth incubated at $37^{\circ} \mathrm{C}$ for 7 days. Blood agar and MacConkey agar incubated aerobically and white chocolate agar incubated anaerobically. Then culture was examined for likely organism by gram staining and specific tests were applied for identification of gram positive and negative bacteria. Whenever no growth seen till 7 th day, blood culture was labelled as sterile.

\section{Antibiotic Sensitivity}

Organisms were isolated in pure culture and subculture in peptone water. It is kept for $24 \mathrm{hr}$. in the incubator to multiply. Agar plates were used for study of bacterial sensitivity disc method. Commercially prepared discs of $6 \mathrm{~mm}$ in diameter were used, antimicrobial content of discs for various antibiotics were ampicillin 10micrgm, cefotaxime $10 \mathrm{micrgm}$, methicillin 5, amikacin $30 \mathrm{~g}$, netilmicin $30 \mathrm{~g}$, erythromycin $5 \mathrm{~g}$, vancomycin $30 \mathrm{~g}$, ciprofloxacin $1 \mathrm{~g}$. Plates were incubated for overnight in air at $35^{\circ} \mathrm{C}-37^{\circ} \mathrm{C}$. The zone of inhibition was measured by millimetre rule. The diameter of clear zone was measured. After measuring the diameter of zone organism was labelled as sensitive, resistant or intermediate by comparing the sizes of zone with control strain. Sensitive labelled when size of zone of the test strain was more than or equal or not smaller than $2 \mathrm{~mm}$. Intermediate was labelled when test zone size was equal to $2 \mathrm{~mm}$, but it was $>3 \mathrm{~mm}$, smaller than controls.

\section{CSF Examination: Routine Microscopy}

A $3-5 \mathrm{ml}$ of CSF was taken in test tube and reported for various features.

Physical appearance: was reported as clear, turbid, haemorrhagic and yellowish. Precipitates were noted by centrifuging the CSF. Glucose determination: were done by glucose oxidase test.

Proteins estimation: were done by spectrophotometers Pandy's test: were done by 2-3ml of phenol with 2-3 drops of CSF in it positive Pandy's were considered when turbidity appears.

Cell count: the count was done in improved Neubauer's chamber by putting 1-2 drops of CSF and 4 drops of CSF diluting fluid in each square. Cell count was done in four corner squares and total count was derived from following formula No. of cells in 4 square x $25=$ total leucocyte count.

\section{RESULTS}

Seventy eight clinically septic cases belongs to $1-2 \mathrm{Kg}$ weight group, which is $78 \%$ of total. Our study shows that $92 \%$ cases have birth weight less than $2.5 \mathrm{~kg} ; 73 \%$ cases were $<34$ weeks gestation while $98 \%$ cases under 37 weeks gestation, which 
shows that late onset septicemia is more common in preterm babies. The ratio of male-to-female is nearly 1.2:1.

Majority of cases (89\%) became culture positive between 3rd-7th day of life.

Prematurity at birth was most common indication of admission, $78 \%$ of cases followed by respiratory distress in $30 \%$, refusal of feed $(15 \%)$, resuscitation at birth (25\%).

$35 \%$ babies stayed in nursery for $10-14$ days while $24 \%$ babies stay for more than 20 days. More than $79 \%$ babies stay in nursery for more than 10 days. Average stay reported during study was 17 days/patient.

Table: 1 shows that main clinical features were lethargy $89 \%$, refusal to feed $84 \%$, jaundice $60 \%$ and hypothermia $63 \%$.

Table: 2 and 3 shows that maximum number (64\%) of values of TLC were within normal limit expected for neonates. While $14 \%$ values of TLC were below the normal count and $22 \%$ values of TLC were above normal reference value. CSF was done in only 13 babies and was positive in 2 cases $(15 \%$ of CSF positive).

A total of 115 cultures were obtained from 100 patients (Table: 4). Most common organism isolated was staph aureus (48.5\%).

Staphylococcus were most sensitive to vancomycin $(69.9 \%)$ followed by amikacin $48 \%$, ciprofloxacin, linezolid gentamicin, piperacillin, cefotaxime, co-trimoxazole in decreasing order. E. coli was most sensitive to amikacin followed by netilmicin, ciprofloxacin, Cefotaxime, gentamicin, piperacillin in decreasing order. Klebsiella was most sensitive to amikacin $(63.1 \%$ cases $)$ followed by netilmicin, ciprofloxacin, piperacillin, co-trimoxazole, cefotaxime, meropenem, gentamycin in decreasing order. Enterococcus were most sensitive to vancomycin $(60 \%)$ followed by linezolid (50\%).

A $67 \%$ cases with nosocomial septicemia were discharged while mortality was reported in $25 \%$ cases. In the study, maximum deaths were occurred in 5-12 days of life.

\section{DISCUSSION}

Out of 100 babies, 55 babies (55\%) were male and 45 babies (45\%) were female indicating a male predominance. This observation is comparable to results by various authors. M.L. Moro reported no gender association of septicaemia. Mortality was high in female neonates. In the present study, it is $44 \%$ and $56 \%$ for male and female neonates respectively.

Incidence of septicemia is high in low birth weight babies. There is direct correlation between septicemia rate and weight group, as weight decreases rate of septicemia increases. In the present study, 92\% babies were below $2.5 \mathrm{Kg}$. This observation was supported by different authors. Heming et al. ${ }^{1}$ found that sepsis was 2.2 times greater in babies with weight $<1500 \mathrm{gm}$ as compared to $>1500 \mathrm{gm}$ babies. In the present study there was only 4 cases of $<1000 \mathrm{gm}$, because most ELBW babies were certified within 3 days. The mortality was also high in low birth weight babies.

Pre-maturity is associated with increased risk of nosocomial sepsis due to decreased immunoglobulin and opsonophagocytosis as reported by Fanor off AA and Krediet TG et al. ${ }^{2}$ Mortality is also high in pre-terms as compared to term neonates. In the present study, it is $69 \%$ in pre-terms. This observation was supported by A. Misallati et al. ${ }^{3}$ who reported 8 deaths out of 8 pre-terms with septicemia and one death out of three term babies.

The clinical features of neonatal sepsis are nonspecific and vague. In this study, most common findings were refusal to feed $(84 \%)$ and lethargy (89\%). Refusal to feed as most common feature was also reported by 0 Batistti. ${ }^{4}$ i.e. $77 \%$ cases and $66.25 \%$ by Abida Malik et al. ${ }^{5}$ In the present study, jaundice was detected in $63 \%$ of cases, but in $77.7 \%$ cases jaundice appears to be physiological while $16.67 \%$ cases were outside the physiological range and $31 \%$ cases of jaundice were of direct hyperbilirubinemia.

Total leucocyte count is highly variable with age and weight of babies, particularly neutrophil count. Total leucocyte count ranges from 5000-20,000. In present study count below 5000 was seen in $14 \%$ cases, while counts above 20,000 were seen in $22 \%$ cases. Beutew et al. ${ }^{6}$ found leucocyte count of no help in the diagnosis of septicemia in premature infants. Thrombocytopenia is known in neonatal septicemia. In the present study, it was reported in $77 \%$ cases. This finding was supported by Easton and Carrigon et al. who reported thrombocytopenia in $47 \%$ and $73 \%$ cases respectively.

Neonatal sepsis carries a high mortality rate. Two-third deaths in infants are due to neonatal sepsis. In this study, death rate was $25 \%$. This is comparable with observations by other authors (20\%-33\%). SRS bulletin 2011. ${ }^{7}$ Somanci M. ${ }^{8}$ Mandira Banerjee et al. ${ }^{9} 38 \%$, A. K. Pahwa et al. $^{10}$ reported $77 \%$ deaths with infection and $14 \%$ deaths without infection, while 0 . Battisti. ${ }^{4}$ reported 29\% and 64\% deaths in 'LOS' and 'EOS' respectively.

Mortality rate also varies with gestational age. In present study, $82 \%$ deaths were of pre-terms. This observation was supported by Abida S. Malik et al. ${ }^{5}$ Death rate also varies according to organism. In present study, around 85\% deaths are due to gram-negative bacilli. In present series case fatality by E. Coli was very high ( $23 \%$ of total E. Coli septicemia cases).

Staphylococcus is the most common organism grown from cultures 33\% (33/100). E. Coli being the 2nd most common organism $21 \%$ followed by Klebsiella (19\%). This is comparable to observation by other workers who showed $\mathrm{E}$. Coli as most common organism, while Choudhary $\mathrm{P}$ and Shirivastava G et al. ${ }^{11}$ reported E. Coli as $10 \%$ of total nosocomial septicemia.

Incidence of Klebsiella in this study was 19\%. Mandira Banerjee et al. ${ }^{9}$ A. K. Pawa. ${ }^{10}$ Adeyemo A. A. ${ }^{12}$ P. Choudhary et al. ${ }^{11}$ reported Klebsiella as most common organism arising as nosocomial sepsis in 70.3\%, 68\%, 27.8\%.

In this study, E. coli most sensitive to amikacin $61 \%$ and least sensitive to cotrimoxazole, sensitivity to netilmicin $52.3 \%$, ciprofloxacin $33.3 \%$ which were most commonly used prophylactically in babies admitted in our nursery.

Klebsiella was most commonly sensitive to amikacin $63 \%$ followed by netilmicin $31.5 \%$, least sensitive to ampicillin and cotrimoxazole each. Mandira Banerjee et al. ${ }^{9}$ recorded Klebsiella sensitivity to various antibiotics as ciprofloxacin $31.5 \%$, piperacillin- tazobact (26\%).

Staph was most commonly sensitive to vancomycin (69.9\%) followed by amikacin (48\%), ciprofloxacin 44.4\%) and linezolid (42.4\%).

\section{CONCLUSION}

This study of 100 neonates regarding clinical, bacteriological risk factors profile in late-onset septicemia reveals that, 
1. Neonatal septicemia is more common in male babies.

2. Pre-term and low birth weight babies are commonly affected.

3. Most of babies became septic in first week of life.

4. Peripheral intravenous cannulas, IV fluids, LBW, increased duration of stay are significant risk factors for late onset septicemia.

5. Increase duration of stay increases risk of sepsis vice versa septicemia increases the duration of stay.

6. LBW, pre-term, respiratory distress at birth are significant conditions associated with late onset septicemia.

7. Lethargy, refusal of feed, hypothermia, gastric residual, pale, jaundice and bleeding diathesis are the common presentation of septicemia.

8. Leukopenia is commonly associated with septicemia as compared to leukocytosis.

9. Thrombocytopenia is important early marker of septicemia.

10. Staph is the most common pathogen followed by E. coli.

11. Mortality rate in nosocomial septicemia is $25 \%$.

12. Mortality rate is higher in female babies as compared to male babies.

13. Mortality rate is high in LBW babies.

14. E. Coli carries high fatality rate.

\section{BIBLIOGRAPHY}

1. Hemming VG, Overall JC, Britt MR. Nosocomial infections in a NICU. Results of fourty one months of surveillance. N Eng Jr med 1976;294:1310-131.

2. Fanaroff AA, Korones SB, Wright LL, Verter J, Poland RL. Incidence, presenting features, risk factors and significance of late onset septicaemia in VLBW babies. The National Institute of Child Health and Human Developmental Research Networks. Paediatric Infections Dis Jr 1998;17(7):593-8.

\begin{tabular}{|c|c|c|c|}
\hline $\begin{array}{l}\text { Sl. } \\
\text { No. }\end{array}$ & Clinical Features & $\begin{array}{l}\text { No. of } \\
\text { Cases } \\
(n=100)\end{array}$ & Percentage \\
\hline 1. & Lethargy & 89 & $89 \%$ \\
\hline 2. & Refusal to feed & 84 & $84 \%$ \\
\hline 3. & Hypothermia & 63 & $63 \%$ \\
\hline 4. & Jaundice & 60 & $60 \%$ \\
\hline 5. & Gastric residual & 46 & $46 \%$ \\
\hline 6. & Mottling & 42 & $42 \%$ \\
\hline 7. & Generalized pallor & 37 & $37 \%$ \\
\hline 8. & $\begin{array}{c}\text { Abdominal } \\
\text { distension }\end{array}$ & 30 & $30 \%$ \\
\hline 9. & Hyperthermia & 22 & $22 \%$ \\
\hline 10. & Seizure episodes & 21 & $21 \%$ \\
\hline 11. & Sclerema & 19 & $19 \%$ \\
\hline 12. & $\begin{array}{c}\text { Bleeding } \\
\text { IV/GI/other }\end{array}$ & 15 & $15 \%$ \\
\hline 13. & Apneic episode & 15 & $15 \%$ \\
\hline 14. & Bulging fontanelle & 2 & $2 \%$ \\
\hline
\end{tabular}

Table 1: Clinical Features of Neonatal Septicemia
3. A Misalliti, S E1-Bagharthy and N Shembesh. Blood Culture proven neonatal septicemia: A review of 36 cases. Ind Jr of Pediatrics. 2000;(6):483-486.

4. Battisti, Ruth Mitchison and Pamela A Davis. Changing blood culture isolates in a referral neonatal intensive care unit. Arch Dis Child 1981;56:775-778.

5. Malik Abida, Hasani SE, Khan HM, Ahmad AJ. Nosocomial infections in newborn. Ind Ped 2001;38:68.

6. Beutow KC, Klein SW and Lane RB: Septicemia in premature infants. Am Jr Dis Child 1965;110:29-41.

7. SRS bulletin 2011: vol.46 no.-1.

8. Somanci N, Ovali F, Akdo Z, Daolu T. Neonatal septicaemia in a neonatal intensive care units. Results of four year. Turkish Journal of Ped 1997;39(2):185-93.

9. Banerjee Mandeera, Sahu K, Bhattacharya S, Bhowmick P: Outbreak of neonatal Septicemia with multidrug resistant Klebsiella pneumonia. Ind J Pedtr 1993;60:2527.

10. Pawa AK, Ramji S, K Prakash and S Thirupuram. Neonatal nosocomial infections: profile and risk factors. Indian paediatrics 1997;34:297-302.

11. Chaudhary P, Shrivastawa G, Aggarwal DS, Saini L, Gupta S: Bacteriological study of neonatal infection, Ind Ped 1975;12(6):459-63.

12. Adeymo AA, Akindele JA, Omokhodian SS. Klebsiella septicaemia osteomyelitis and septic arthritis in neonates in Ibadan Nigeria. Annals of Tropical Ped 1993;13(3):285-90.

\begin{tabular}{|c|c|c|c|}
\hline $\begin{array}{c}\text { Sl. } \\
\text { No. }\end{array}$ & TLC & $\begin{array}{c}\text { No. of Cases } \\
(\mathbf{n = 1 0 0})\end{array}$ & Percentage \\
\hline 1. & $<4999$ & 14 & $14 \%$ \\
\hline 2. & $5000-9999$ & 34 & $34 \%$ \\
\hline 3. & $\begin{array}{c}10000- \\
19999\end{array}$ & 30 & $30 \%$ \\
\hline 4. & $>20000$ & 22 & $22 \%$ \\
\hline \multicolumn{4}{|r|}{ Table 2: Showing Distribution of Cases According to TLC } \\
\hline
\end{tabular}

\begin{tabular}{|c|c|c|c|}
\hline $\begin{array}{c}\text { Sl. } \\
\text { No. }\end{array}$ & $\begin{array}{c}\text { Thrombocyte } \\
\text { Count }\end{array}$ & No. of Cases & Percentage \\
\hline 1. & $<20000$ & 2 & $2 \%$ \\
\hline 2. & $20000-49999$ & 18 & $18 \%$ \\
\hline 3. & $500000-99999$ & 42 & $42 \%$ \\
\hline 4. & 1 Lac- 1.5 Lac & 15 & $15 \%$ \\
\hline 5. & $>1.5$ Lac & 23 & $23 \%$ \\
\hline \multicolumn{3}{|c|}{ Table 3: Showing Distribution of Cases According to } \\
Thrombocyte Count \\
\hline
\end{tabular}




\begin{tabular}{|c|c|c|c|}
\hline $\begin{array}{c}\text { Sl. } \\
\text { No. }\end{array}$ & Organism & $\begin{array}{c}\text { No. of Cases } \\
(\mathbf{n = 1 0 0 )}\end{array}$ & Percentage \\
\hline 1. & Staph & 33 & $33 \%$ \\
\hline 2. & Sterile & 32 & $32 \%$ \\
\hline 3. & E. Coli & 21 & $21 \%$ \\
\hline 4. & Klebsiella & 19 & $19 \%$ \\
\hline 5. & Enterococcus & 5 & $5 \%$ \\
\hline 6. & Pseudomonas & 2 & $2 \%$ \\
\hline 7. & Streptococcus & 2 & $2 \%$ \\
\hline 8. & Proteus & 1 & $1 \%$ \\
\hline \multicolumn{3}{|c|}{ Table 4: Showing Distribution of Cases According to } \\
Blood Culture Pattern of Septicemia \\
\hline \multicolumn{4}{|c|}{}
\end{tabular}

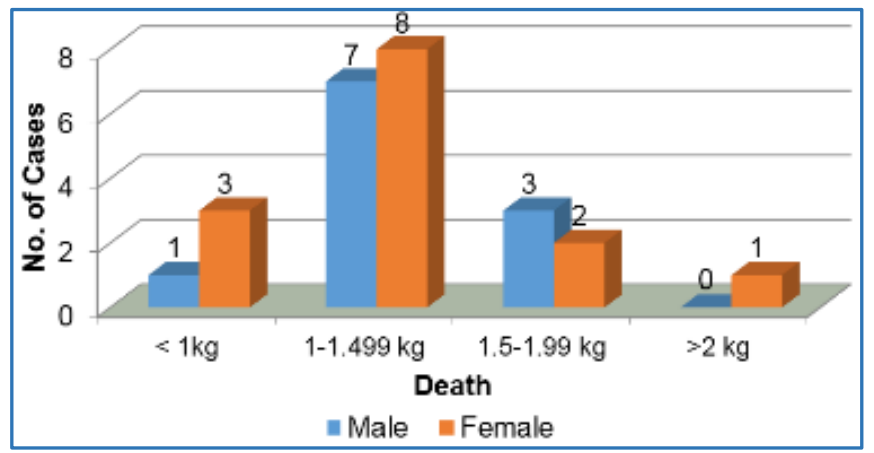

Death Distribution According To Weight and Sex 